Manuelle Medizin 2011 · 49:225

DOI 10.1007/s00337-011-0861-8

(c) Springer-Verlag 2011

\author{
L. Beyer \\ DGMM Ärztehaus Mitte, Jena
}

\title{
Manuelle Medizin - Erfolge in der Praxis, aber Forschungsbedarf
}

Liebe Leserinnen, liebe Leser,

bevor ich Ihnen die Beiträge dieses Hefts vorstelle, möchte ich den Kollegen aus zwölf Kliniken gratulieren, die sich zu der Arbeitsgemeinschaft nichtoperativer orthopädischer Akutkliniken (ANOA) zusammengeschlossen haben. Sie stellen sich, ihre manualmedizinischen Kliniken und ihre Ziele seit kurzer Zeit unter www.anoa-kliniken.de vor. Hier ein Zitat aus dem Beginn eines Gesprächs mit dem Patienten:

„Sie wollen sich trotz der Empfehlung Ihres Arztes, einen operativen Eingriff vorzunehmen, über alternative und risikoärmere Behandlungen informieren und eine Zweitmeinung einholen? Wir bieten sanfte Methoden, zum Beispiel manuelle Medizin, osteopathische, naturheilkundliche Verfahren und andere.“

Fast im Gegensatz zu diesem klinischen Praxiserfolg findet Ammer in allen seinen kommentierten Auszügen neuer relevanter Literatur: „... aufgrund der geringen Studien- und Patientenzahl wird die Evidenz der Wirksamkeit von Manualtherapie auf Schmerz und Körperfunktion von Patienten mit ... [siehe Beiträge in diesem Heft] ... als nicht eindeutig beurteilt."

Vorläufige Ergebnisse von Alt u. Alt sollen uns ermutigen, weitere Erfahrungen mit der Anwendung der Sonographie in der manuellen Medizin im Kontext osteopathischer viszerofaszialer Techniken zu sammeln, sowohl als begleitende Diag- nostik wie auch zu didaktischen Zwecken bei viszerofaszialen Behandlungen.

Rohde sucht in der Evolution der Aufrichtung von Fuß und Hand des Menschen nach möglichen Ansätzen manualtherapeutischen und naturheilkundlichen Vorgehens.

Valet et al. beschreiben somatosensorische, affektive, kognitive, vegetative und motorische Aspekte aus Untersuchungen zur zerebralen Verarbeitung von Schmerzen mithilfe der funktionellen Bildgebung. Die funktionelle Bildgebung erlaubt die nichtinvasive Untersuchung am Menschen in vivo und bei vollem Bewusstsein. Ein einzelnes, klar abgegrenztes „Schmerzzentrum“ existiert nicht. Eine Vielzahl von Hirnregionen trägt zu den verschiedenen Aspekten der Schmerzverarbeitung bei, und ihre Interaktion führt zum komplexen Sinneseindruck „Schmerz“. Durch diese Forschung besteht die Hoffnung, dass in naher $\mathrm{Zu}$ kunft auch therapeutische Konsequenzen, zum Beispiel im Sinne eines Therapiemonitorings oder Neurobiofeedbacks durch funktionelle Magnetresonanztomographie (fMRT) in Echtzeit, abgeleitet werden können.

Buchmann et al. komplettieren ihre Zusammenstellung manualmedizinischer Syndrome durch die des Thorax und des Abdomens - Befundhäufungen aus dem muskuloskeletalen und viszeralen System. Es gibt durchaus einige kritische Stimmen, die den Sinn einer Aufstellung so vieler Syndrome bezweifeln. Die Zeit ist aber reif, die kollektive Erfahrung aus der täglichen Praxis der Manualmedizin in einem zusammenfassenden Überblick der erhobenen Befunde - Befunde, die nur manuell mit der Hand und unter funktionellem Aspekt erhoben werden - für sich selbst und für die anderen medizinischen Fachgebiete darzustellen. Die manualmedizinische Diagnostik kann einen wertvollen Beitrag in der Abgrenzung von struktureller und funktioneller pathologischer Veränderung leisten.

Man möchte fast meinen, wir sind nur so gut, wie wir in der Lage sind, unsere Arbeit selbst darzustellen. Wie immer: Auch Ihr Beitrag und Ihre Erfahrung sind dazu gern willkommen.

Mit freundlichen Grüßen

Ihr

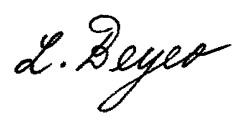

L. Beyer

Korrespondenzadresse

Prof. Dr. L. Beyer

DGMM Ärztehaus Mitte

Westbahnhofstr. 2, 07745 Jena

lobeyer@t-online.de 